Dom. Cien., ISSN: 2477-8818

Vol. 4, núm. 1, enero, 2018, pp. 131-140

El marketing y la fidelización empresarial como apuesta estratégica para pymes en Ecuador

DOI: $10.23857 /$ dc.v4i1.727

Número Publicado el 15 de enero de 2018

Ciencias económicas y empresariales

Artículo de investigación

\title{
El marketing y la fidelización empresarial como apuesta estratégica para pymes en Ecuador
}

Marketing and business loyalty as a strategic bet for SMEs in Ecuador

Marketing e lealdade empresarial como aposta estratégica para as

PMEs no Equador

Cesar R. Alarcón-Chávez ${ }^{\mathrm{I}}$

cesaracho@hotmail.com

Marco I. Granda-García II

marcograndag@hotmail.com

Recibido: 24 de junio de 2017 * Corregido: 28 de octubre de 2017 * Aceptado: 05 de diciembre de 2017

I. Magister en Gerencia Educativa, Diplomado Superior en Evaluación Institucional Universitaria, Economista, Docente de la Universidad Laica Eloy Alfaro de Manabí, Manta, Ecuador.

II. Ingeniero en Ciencias Empresariales con Especialización en Gestión Empresarial, Master Universitario en Información Digital, Especialidad en Buscadores: Marketing Online (SEM) y Posicionamiento WEB (SEO), Docente de la Universidad Laica Eloy Alfaro de Manabí, Manta, Ecuador. 


\section{Resumen}

La globalización ha transformado los enfoques tradicionales de comercialización y crecimiento empresarial. Las pymes en Ecuador representan el entramado industrial, más numeroso del país ya que aglutinan a más del $97 \%$ b de las empresas. Sin embargo, la complejidad de un mercado constantemente en cambio, y con clientes cada vez más exigentes, las obliga a emplear el marketing como herramienta para establecer vínculos de tipo subjetivo con los clientes, sobre la base de la satisfacción de necesidades, demandas de servicios y productos de calidad. El marketing contempla un vasto campo de acción, pero en las actuales circunstancias la fidelización empresarial se debe establecer como un punto de partida, para un cambio de cultura por parte del empresariado y la empresa, que facilite la tarea de hacer frente a la incertidumbre. La producción de valor subjetivo es la idea de toda campaña de fidelización, orientada hacia el cliente y el marketing, pensado para pymes puede significar una victoria en este sentido.

Palabras clave: marketing; pymes; fidelización.

\section{Abstract}

Globalization has transformed traditional marketing and business growth approaches. SMEs in Ecuador represent the industrial framework, which is the most numerous in the country, since they account for more than $97 \%$ of companies. However, the complexity of a constantly changing market, and with increasingly demanding customers, forces them to use marketing as a tool to establish subjective links with customers, based on the satisfaction of needs, demands for services and quality products. Marketing contemplates a vast field of action, but in the current circumstances, corporate loyalty must be established as a starting point for a culture change by business and the company, which facilitates the task of facing uncertainty. The production of subjective value is the idea of any loyalty campaign, oriented towards the client and marketing, thought for SMEs can mean a victory in this sense.

Keywords: marketing; SMEs; loyalty

\section{Resumo}

A globalização transformou as abordagens tradicionais de marketing e crescimento de negócios. As PME no Equador representam o quadro industrial, que é o mais numeroso do país, pois representam 
mais de $97 \%$ das empresas. No entanto, a complexidade de um mercado em constante mudança e com clientes cada vez mais exigentes obriga-os a usar o marketing como uma ferramenta para estabelecer vínculos subjetivos com os clientes, com base na satisfação das necessidades, demandas por serviços e produtos de qualidade. O marketing contempla um vasto campo de ação, mas nas circunstâncias atuais, a lealdade corporativa deve ser estabelecida como ponto de partida para uma mudança de cultura por parte dos negócios e da empresa, o que facilita a tarefa de enfrentar a incerteza. A produção de valor subjetivo é a idéia de qualquer campanha de fidelidade, orientada para o cliente e marketing, o pensamento para as PMEs pode significar uma vitória nesse sentido.

Palavras chave: marketing; PME; lealdade

\section{Introducción}

La economía del Ecuador se estructura fundamentalmente sobre empresas de pequeño y mediano tamaño; una cifra que destaca a simple vista y que viene dada por el hecho de que en el país, para el año 2016 existían alrededor de 845.745 empresas de diverso tipo. No obstante, para ese año el 90,5\% de estas se pueden clasificar como microempresas, cuyas ventas anualizadas estaban por debajo de los 100.000 dólares al año; mientras que el 7,5\% del universo empresarial, pertenece a la categoría de pequeña empresa, con ventas anuales entre los 100.000 y 1.000 .000 de dólares. (INEC, 2016). Una de las características más notables de las microempresas y de las pequeñas empresas, radica precisamente, en la capacidad para generar empleo, más; sin embargo, por tratarse de pequeñas unidades de producción no se debe desconocer que son mucho más permeables a los cambios y fluctuaciones del mercado y la economía.

En consecuencia, si se quiere hablar de crecimiento y desarrollo de manera acertada en el Ecuador, no debe obviarse la configuración actual del entramado económico que lo compone. Avanzar hacia la senda del crecimiento, implica entonces hablar de emprendedores, de unidades autónomas, que no solo luchan por abrirse paso, sino que llevan adelante sueños de posicionarse a todos los niveles en un mercado caracterizado por la incertidumbre y la competencia.

Una realidad cada vez más compleja y difícil para los "pequeños" requiere de nuevas maneras de actuar, de pensar y de hacer las cosas en el plano económico. En este sentido el marketing; pensado inicialmente para las grandes empresas, puede y de hecho requiere ser adaptado a los requerimientos 
de las pymes en Ecuador, a objeto de que estas no queden finalmente a la zaga de las dinámicas competitivas con que deben enfrentarse día a día.

Las pymes están experimentando cambios, la preocupación por el negocio ha ido posicionándose en la conciencia del pequeño empresario y de allí la fuerte apuesta por este tipo de herramientas de dirección y gestión empresarial, de posicionamiento comercial, de fidelización o de planeación que brinda el marketing en ésta área, ya que incluso, puede llegar a significar la ubicación de productos fuera de las fronteras nacionales si se le emplea inteligentemente.

El cambio de época se está dando irremediablemente y junto a este, las pymes deben adaptarse rápidamente. La era de la comunicación y la tecnología, de las redes y la comunicación en tiempo real imponen el ritmo. Así el marketing, el marketing pensado para pymes, cobra especial importancia para hacer frente al fenómeno de la globalización. Por todo ello las pequeñas empresas ecuatorianas no pueden desaprovechar las oportunidades que ofrecen la nueva economía y un consumidor cada vez más exigente. La adaptabilidad debe constituirse en un principio de trabajo para las pymes.

Este trabajo es de tipo bibliográfico y realiza una revisión de textos y literatura especializada, respecto al marketing empresarial, para de allí trazar algunas líneas que sirvan de base para su aplicación rigurosa en las pymes del Ecuador.

\section{La evolución del marketing}

El marketing cambia constantemente como teoría, para poder dar respuestas a los consumidores. La satisfacción del cliente comienza a verse en este sentido, como un aspecto clave para el crecimiento sostenido. La transparencia, la cercanía y la comunicación efectiva se han venido imponiendo como una variable ética de peso, en las relaciones de intercambio comercial y vivencial entre el cliente y la empresa. Todo esto ha superado a las concepciones iniciales del marketing, incluso Ortiz (2018) establece que el marketing tradicional:

Se refiere a todas aquellas prácticas, que los especialistas de la mercadotecnia emplearon en la mitad del siglo pasado e inicios del actual. El marketing nació como una herramienta para ayudar a la producción en una era donde el fin primordial, era vender productos más allá de ofrecer un valor intangible. 
Este apartado resulta pertinente, puesto que sintetiza toda una tradición en la historia del marketing, dado que abunda la literatura que lo comprende, como una palanca de las grandes empresas y multinacionales. Pero nada más lejano de la realidad. El marketing, ofrece un sinnúmero de ventajas para la proyección económica empresarial de las pymes, la captación de clientes, y la planificación pormenorizada de estrategias que capitalicen fidelidad, hacia una marca o un producto determinado. La eficacia del marketing no obedece a cuestiones de tamaño, sino que depende del entendimiento de la dinámica económica en la cuan se inscribe como teoría aplicable.

Por otra parte, Velázquez (2015), define que el marketing empresarial puede definirse como el que se realiza para que una empresa logre vender sus productos o servicios a otras empresas, que los usan a su vez para fabricar sus propios productos o apoyar sus operaciones diarias.

De hecho, cuando se habla de marketing para pymes la intención gira en torno a la posibilidad de dar respuestas en las principales áreas del marketing a la pequeña empresa o unidad de negocios. Toda vez que sea posible transmitir efectivamente cómo el marketing, es realmente una disciplina con un gran peso estratégico, científico y metodológico; una poderosa herramienta para mejorar la imagen de marca e incrementar las ventas. Por consiguiente, realizar un buen marketing conlleva buenos resultados empresariales. El buen marketing sin duda, es la clave para la competitividad empresarial, que a su vez deviene en el activador fundamental de la demanda y el motor de la economía ecuatoriana.

\section{La fidelización del cliente como principal apuesta estratégica de las pymes en Ecuador}

En un entorno competitivo, con clientes cada día más exigentes y un escenario económico cambiante, el posicionamiento de la marca y la fidelización de los clientes, cobran cada día mayor fuerza como garantía de éxito para las pequeñas empresas. En el caso ecuatoriano, esta alternativa resulta totalmente válida dada la similitud de los productos y servicios, ofrecidos por las microempresas y las pequeñas empresas. La fidelización, cuya base de acción se halla en la política de acercamiento y de servicios que pueda ofrecer permanentemente al consumidor, sin duda constituye el fundamento de toda política microempresarial. Por lo tanto, un mejor servicio, que brinde respuestas oportunas a los clientes, amplían las posibilidades de éxito en los mercados actualmente. Saber vender en el siglo XXI, para las pymes es una tarea aún por construir. 


\section{La fidelización de clientes para las pequeñas empresas}

Según Bernués y Alcaide (2013), en cuanto a la relevancia de la fidelización establecen que:

Las Pymes se enfrentan a una coyuntura compleja, un cambio de época en el que será fundamental la capacidad que tengan de orientar el negocio hacia el cliente. Personalizando y adecuando sus servicios a esos clientes. Clientes que es preciso gestionar de la forma adecuada, con idea de que permanezcan muchos años haciendo negocios con la compañía. El objetivo de cualquier empresa debe ser la captación y el mantenimiento de clientes rentables a lo largo del tiempo con objeto de maximizar el beneficio de la Pyme.

Para ello es vital orientar la organización y las personas que la componen hacia esos clientes, lo que nos permitirá identificar claramente sus necesidades y deseos, con objeto de ofrecerles productos que las satisfagan. Sólo si los clientes están satisfechos y retenidos serán fieles a la compañía,(p.11).

La captación y la permanencia del cliente vienen a representar dos aristas de una misma ecuación, en el enfoque que las pymes deben asumir dentro de un mercado cambiante y caracterizado por la incertidumbre; no obstante, la satisfacción viene a cerrar el ciclo que se inicia con la captación en la fidelización, pero esta vez, adaptada a los nuevos enfoques que el marketing ha venido desarrollando a lo largo del tiempo. Por lo tanto, fidelizar no es más que impulsar un proceso prensado para garantizar la construcción de un vínculo entre el cliente y la empresa. Una relación subjetiva de carácter permanente. Recordemos que el marketing se fundamente en la generación y estímulo de emociones en los sujetos objeto de la estrategia comercial que impulsa; es decir, que busca premeditadamente la generación de valor subjetivo.

El valor subjetivo (VS), se refiere a la capacidad que tiene una empresa de usar connotaciones asociadas a su imagen para influir en el comportamiento de los clientes. El valor subjetivo es muy importante para empresas que no pueden diferenciarse por el valor objetivo. Generalmente, son firmas que ofrecen productos genéricos (commodity), o que compiten en industrias en las cuales nuevos actores o los existentes pueden introducir productos con mejor desempeño y más baratos (clase ejecutiva, 2012).

En este contexto, Bernués y Alcaide (2013) determinan que: 
Para ello serán claves una serie de pautas, que la organización tendrá que desarrollar y que facilitarán la tarea de fidelizar.

La orientación de la compañía hacia el Cliente.

La implantación de una política de marketing relacional.

El desarrollo de continuas acciones que doten la relación de contenidos de valor.

Tratar de generar un atractivo emocional para nuestros clientes.

Lo que falta se inventa. Creatividad e innovación para paliar los presupuestos ajustados en la pequeña empresa.

Es importante apuntar también lo siguiente:

Fidelizar es establecer un diálogo continuo con el cliente

Fidelizar es pensar a largo plazo y conocer a fondo a los clientes.

Fidelizar es combinar los beneficios a corto plazo para el cliente con beneficios a largo plazo.

Fidelizar es mezclar lo racional con lo emocional.

Fidelizar es buscar formas de aportar un mayor valor a nuestros clientes.

Como puede observar las pymes en el Ecuador, deberán asumir por si mismas la construcción de toda una nueva cultura de trabajo, una nueva manera de modelar las estrategias de mercado y comprender a fin de cuentas que el mundo ha cambiado con la revolución tecnológica y la globalización.

En el país estas empresas son la representación mayoritaria del conglomerado industrial que lo compone, además de representar la mayor fuente de empleo. Carvajal, Solís, Villamar y Hermida (2017) consideran que:

Las ciudades del Ecuador, tienen un acelerado crecimiento y desarrollo en lo concerniente a las Pymes; sin embargo, estas deben enfrentar la competencia de grandes empresarios con poder económico, que provienen de ciudades grandes, quienes se diferencian por su alto nivel de 
El marketing y la fidelización empresarial como apuesta estratégica para pymes en Ecuador

productividad el cual es alcanzado por la correcta aplicación de la administración de operaciones en sus procesos, aspecto que las pone en desventaja.

Las Pymes son fuente generadora de empleo en el Ecuador, es necesario que las mismas logren alcanzar un desarrollo sostenible en el tiempo a través de procesos y productos de calidad.

Toda posibilidad de superación de las debilidades que padecen actualmente las pymes, depende de las estrategias administrativas que empleen. Pero acaso no sería acertado preguntarse ¿el marketing para pymes no es un complemento del área administrativa, de ventas y de mercadeo de la empresa?, ¿puede acaso el inmenso sector empresarial que (representado en el pequeño empresariado nacional del Ecuador), seguir desestimando las herramientas que la globalización y la ciencia le brindan? Para todas estas preguntas, la respuesta más idónea tiene que ver con la aceptación de la necesidad de la adopción y adaptación del marketing a las necesidades de las pymes para enfrentar los retos actuales.

\section{Conclusiones}

La teoría del marketing empresarial es acertada, cuando busca establecer vínculos subjetivos con los clientes; sin embargo, el enfoque tradicional que proyecta su uso en las grandes cadenas de comercialización debe quedar en el pasado. Las pymes representan el núcleo económico fundamental del Ecuador, debido a la cantidad y deben contar con herramientas científicas desde las cuales apalancar los mercados en los que interactúan a diario.

La fidelización, es el primer camino a transitar para un cambio profundo en la manera en que las pymes, deben concebir el negocio. El establecimiento de vínculos permanente con el cliente, sin duda elevara la calidad de los productos y servicios que estas ofrecen, en vista de la importancia que tiene la satisfacción y la opinión de éste para el éxito de la empresa.

El hecho de comenzar por la comprensión de la relevancia, que en la actualidad adquiere la elaboración de una campaña de fidelización asertiva y bien fundamentada no exime a las pymes de la adopción de estrategias de comercialización basadas en las redes sociales u otras alternativas igualmente válidas.

Un crecimiento económico más acelerado en el Ecuador, depende del crecimiento de las pymes, pero estas tienen necesariamente que comprender que el marketing para pymes, constituye una poderosa 
herramienta para la superación de las dificultades actuales que experimentan en un entorno cambiante y de constante incertidumbre.

La fidelización en el futuro cercano será un punto de partida incuestionable, para estrechar lazos permanentes entre los consumidores y los prestadores de servicios, sólo queda desarrollar nuevos enfoques de marketing para las pequeñas empresas que sueñan a diario con crecer de manera sostenida en nuestro país.

\section{Referencias Bibliográficas}

Bernués y Alcaide, Marketing para Pymes (2013). Recuperado de www. marketingypymesebook.com Carlos Yance Carvajal, Luis Solís Granda, Ivonne Burgos Villamar y Lia Hermida Hermida (2017): "La importancia de las PYMES en el Ecuador", Revista Observatorio de la Economía Latinoamericana, Ecuador, (junio 2017). Recuperado de http://www.eumed.net/cursecon/ecolat/ec/2017/pymes-ecuador.html

Instituto Nacional de Estadística y Censos (2016), Ecuador registró 843.745 empresas en 2016. Recuperado de http://www.ecuadorencifras.gob.ec/ecuador-registro-843-745-empresas-en-2016/

Marketing: La marca y el valor subjetivo (2012) http://www. claseejecutiva.cl/2012/06/marketing-lamarca-y-el-valor-subjetivo/

Mediasource, Marketing tradicional (2017). Recuperado de https://www.mediasource.mx/ blog/marketing-tradicional

Marketing4ecommerce, Marketing empresarial: definición y conceptos importantes (2015). Recuperado de https://marketing4ecommerce.mx/marketing-empresarial-definicion-y-conceptosimportantes/ 